\title{
Synaptic Plasticity in the Rat Supraoptic Nucleus during Lactation Involves GABA Innervation and Oxytocin Neurons: A Quantitative Immunocytochemical Analysis
}

\author{
Ursula Gies ${ }^{1}$ and Dionysia T. Theodosis ${ }^{2}$ \\ 'Department of Anatomy and Cytobiology, Justus Liebig University, W6300 Giessen, Germany and INSERM U.378, \\ Neuroendocrinologie Morphofonctionelle, Université de Bordeaux II, 33076 Bordeaux, France
}

The hypothalamic supraoptic nucleus (SON) is known to undergo synaptic remodeling in response to physiological stimuli, such as lactation. We here investigated the involvement of the GABAergic innervation in such plasticity by carrying out comparative ultrastructural analyses after postembedding immunogold labeling for GABA on sections of the SON from virgin and lactating rats.

Using random single-section analysis to estimate the numerical density of synapses (from areal measurements of the anterior, mid, and posterior portions of the nucleus), we found that the overall density in either group was about 35 $\times 10^{6}$ synapses $/ \mathrm{mm}^{3}$, of which over one-third were GABA immunoreactive. The GABAergic terminals formed mainly symmetrical synaptic contact on neurosecretory somata and dendrites; the density of axodendritic contacts was twice that of axosomatic contacts. Despite hypertrophy of the neurosecretory neurons in lactating rats, the overall synaptic density did not diminish and was similar to that evaluated in virgin rats. When we estimated synaptic densities in the neuropil (by subtracting the proportion of sampled areas occupied by soma profiles), we found a statistically significant increase in the density of GABAergic synapses, in particular in the mid and posterior portions of the SON in lactating animals. On the other hand, the density of GABAimmunonegative synapses did not differ in the two groups. Terminals contacting more than one postsynaptic element in the same plane of section ("shared" synapses) were visible in the nuclei of virgin and lactating rats and about half in each group were GABAergic. They were comparatively rare but their incidence doubled in lactating animals. Lastly, in a separate analysis of ultrathin sections where we identified both pre- and postsynaptic elements by simultaneous dual immunogold localization of GABA and oxytocin-neurophysin (OT-Np) immunoreactivities, we found that in virgin rats, over one-third of all synapses on either oxytocinergic

\footnotetext{
Received Jan. 5, 1993; revised Oct. 14, 1993; accepted Oct. 26, 1993.

We thank Professor A. Oksche for his constant support and encouragement, Drs. D. A. Poulain and D. Voisin for their suggestions and reading of the manuscript, and Mr. S. Senon for his photographic expertise. This study was partially supported by fellowships to U.G. from the Deutsche Forschungs Gemeinschaft (NU 36/2-3) and European Science Foundation (STF/2890) and a grant to D.T.T. from the Conseil Régional d'Aquitaine (9103059).

Correspondence should be addressed to Dr. D. T. Theodosis, INSERM U.378, Neuroendocrinologie Morphofonctionelle, Université de Bordeaux II, 146 rue Léo Saignat, F-33076 Bordeaux, France.

Copyright @ 1994 Society for Neuroscience $0270-6474 / 94 / 142861-09 \$ 05.00 / 0$
}

or OT-Np-negative (presumptive vasopressinergic) somatic profiles were GABA immunoreactive. In lactating rats, this proportion increased significantly on oxytocinergic profiles but not on OT-Np-negative profiles. Our observations thus provide further evidence for physiologically linked synaptic plasticity in the SON and indicate that this plasticity affects GABAergic terminals making single or "shared" synapses on oxytocinergic neurons.

[Key words: hypothalamus, oxytocin, GABA, immunocytochemistry, morphometry, electron microscopy, lactation]

The supraoptic (SON) and paraventricular (PVN) nuclei of the hypothalamus, which contain the magnocellular ncurons secreting the neurohormones oxytocin and vasopressin, undergo a synaptic and neuronal-glial plasticity intimately linked to physiological stimuli that result in increased oxytocin release (parturition, lactation, prolonged dehydration) (for review, see Theodosis and Poulain, 1987, 1993; Hatton, 1990). Although oxytocin and vasopressin neurons occur intermingled in about equal proportions in these nuclei, we have established in several analyses that this structural plasticity affects predominantly the oxytocinergic system (Chapman et al., 1986; Theodosis et al., 1986a,b; Montagnese et al., 1987; Theodosis and Poulain, 1989).

Until now, synaptic remodeling in the magnocellular nuclei has been appreciated because of reversible changes in the incidence of a particular synaptic configuration, that of axonal terminals synapsing onto two or more postsynaptic elements in the same plane of section ("shared" synapses). Such terminals are rare in the nuclei of animals under basal hormone release but become more frequent in stimulated animals (Theodosis et al., 1981; Hatton and Tweedle, 1982; Theodosis and Poulain, 1984; Tweedle and Hatton, 1984). Nevertheless, the morphometric analyses performed until now have not permitted determination of the full extent of synaptic rearrangements in these nuclei. Also, the identity of the afferent input involved in such plasticity has not been clearly established.

Hypothalamic magnocellular neurons are richly innervated by GABA synapses (Van Den Pol, 1985; Theodosis et al., 1986c; Buijs et al., 1987; Decavel and Van Den Pol, 1990). In earlier light microscopic studies, where we analyzed semithin sections immunostained for glutamic acid decarboxylase (GAD) and oxytocin, we estimated that about half the synapses on oxytocinergic or vasopressinergic neurons in the SON contain this neurotransmitter. Moreover, our electron microscopic observations showed that many "shared" synapses in the nuclei of lactating rats were GABA immunoreactive, which suggested 
that the GABAergic input to the nucleus was involved in its synaptic rearrangements (Theodosis et al., 1986c).

The present study was undertaken to analyze further the GABAergic innervation of magnocellular neurons and to determine its contribution to synaptic plasticity. We thus carried out a comparative synaptic density analysis of ultrathin sections of the SON from virgin and lactating rats stained with a postembedding immunogold procedure for GABA. We also analyzed sections that underwent dual immunogold staining for GABA and oxytocin-related neurophysin (OT-Np) to determine the relative importance of such innervation on each of the neurosecretory systems. We chose the SON for study because it is a quite homogeneous neuronal center, being composed cssentially of magnocellular neurons and their processes, glial cells that are in the main astrocytes (see, e.g., Bonfanti et al., 1993), and blood vessels.

\section{Materials and Methods}

Tissue preparation. The tissue examined in this study was derived from three virgin female and three lactating Wistar rats raised in our own colony and maintained in a controlled environment with food and water ad libitum. The latter had nursed litters of 10 pups for $11 \mathrm{~d}$. Under sodium pentobarbital anesthesia $(50 \mathrm{mg} / \mathrm{kg}$, i.p.), the animals were perfused intracardially first with a heparinized saline solution (5000 IU/ liter heparin) and then with $3 \%$ glutaraldehydc in $0.1 \mathrm{M}$ sodium cacodylate buffer. Blocks containing the anterior, mid, and posterior portions of the SON, corresponding roughly to stereotaxic coordinates A 8.20$8.08,7.70-7.60$, and $7.20 \mathrm{~mm}$, respectively (Paxinos and Watson, 1986), were dissected from frontal slices of each brain cut on a vibratome $(200$ $\mu \mathrm{m})$. They were osmicated in $2 \% \mathrm{OsO}_{4}$ in Millonig's buffer, dehydrated in a graded series of ethanol, and embedded in Epon. Ultrathin sections were cut with an ultramicrotome and collected on uncoated 300 mesh nickel grids.

Immunocytochemistry. For immunostaining, we used a postembedding immunogold procedure described in detail in (Theodosis et al., $1986 \mathrm{a}, \mathrm{c}$ ) with a rabbit polyclonal serum raised against GABA (gift of $M$. Geffard, INSERM, Bordeaux, France) and mouse monoclonal antibodies raised against OT-Np (gift of $\mathrm{H}$. Gainer, NIH, Bethesda, MD); the production and specificities of the antibodics are described in detail in Seguela et al. (1984) and Ben-Barak et al. (1985), respectively. Briefly, for single GABA immunostaining, the ultrathin sections were first treated with a saturated solution of sodium metaperiodate (15-30 min), rinsed, placed in $10 \%$ normal goat serum for $1 \mathrm{hr}$ and then in antiGABA serum (diluted 1:1000) for $24 \mathrm{hr}$ at $4^{\circ} \mathrm{C}$; immunoreactivity was revealed with $10 \mathrm{~nm}$ colloidal gold particles coupled to anti-rabbit immunoglobulins (GAR 10, Janssen; diluted 1:16). For double labeling, the grids were incubated in a mixture of primary antibodies (anti-GABA diluted 1:1000 and anti-OT-Np diluted 1:50) for $48 \mathrm{hr}$ at $4^{\circ} \mathrm{C}$ and then in a mixture of GAR 10 and $5 \mathrm{~nm}$ gold particles coupled to anti-mouse immunoglobulins (GAM 5; final dilutions, 1:16 and 1:5, respectively). All antibodies were diluted in Tris-buffered saline (TBS) containing $0.25 \%$ bovine serum albumin (BSA); each incubation step was followed by a thorough rinse in TBS with 10\% BSA. The sections were contrasted with uranyl acetate and lead citrate before examination with a Philips 201 and CM10 electron microscopes.

Quantitative analysis. Quantification was performed on electron micrographs of immunostained sections that included the whole coronal span of each portion of the SON (20-40 micrographs per block, three blocks per animal, three animals per group); for each block, there was an equal proportion of photographs that included the ventral (including the ventral glia lamina), middle, and dorsal aspects of the nucleus. The areas photographed were selected in a systematic random fashion with reference to their position in the grid space (see Weibel and Bolender, 1973); the investigator was unaware of the experimental conditions while taking the pictures and during the analysis. The photographs were taken at an original magnification of $8000 \times$; they were further magnified to $25,000 \times$ by printing. In cach micrograph, we counted all synapses showing pre- and postsynaptic (PSD) densities and at least three vesicles apposed to a density (a total of 618 and 681 synapses were thus counted in virgin and lactating rats, respectively). We also noted whether the synapses contained GABA immunoreactivity (see Results). For each synapse, we measured the trace length of the PSD with a calibrated magnifying glass; the total area and the area occupied by all neuronal soma profiles in each micrograph were measured with an MOP-AM O2 (Kontron). The numerical density of synapses was calculated according to the formula $N_{\mathrm{v}}=N_{A} / \bar{D}$, where $N_{A}$ corresponded to the number of synaptic contacts per unit area and $\bar{D}$ the mean trace length of PSD (Weibel and Bolender, 1973). In estimating the area of synaptic contacts we assumed that synaptic disks were circular in shape and that the diameter of disks was equal to their mean trace length (see also Colonnier and Beaulieu, 1985). It should be noted that mean PSD lengths in the SON of virgin $(515.6 \pm 258.1 \mathrm{~nm}$, mean $\pm \mathrm{SD})$ and lactating (470.9 $\pm 237.6 \mathrm{~nm}$ ) rats were not significantly different (Kolmogorov-Smirnov test); the data werc pooled to give an overall mean length of $492.8 \pm$ $248.7 \mathrm{~nm}$, corrected to the "true" mean trace length of $605.5 \mathrm{~nm}$ by the semigraphical method recommended by Weibel and Bolender (for further details, see Weibel and Bolender, 1973).

In these photographs, we also counted the number of axonal terminals making synaptic contact onto two or more postsynaptic elements in the same plane of section ("shared" synapses; see Results) and recorded whether they were or were not GABA immunoreactive.

The analysis of double-stained sections was carried out by counting directly on grids in the electron microscope, using a random sampling procedure (for further details, see Theodosis et al., 1986a). Briefly, within each selected grid space, all profiles of neurosecretory somata were counted. The plasmalemma of each soma profile was then followed to determine the number of synapses it received and whether they were or not GABA immunoreactive; the cytoplasm of the selected soma was then examined to see whether it contained peptide immunoreactivity (see Results). A total of 51 soma profiles, of which 28 were OT-Np positive, were examined in virgin rats; in lactating rats, we examined 39 soma profiles, of which 19 were OT- $\mathrm{Np}$ positive. They were derived from three different sections of the mid-portion of the SON from each of three rats per group.

\section{Results}

\section{Single immunostaining for $G A B A$}

Many GABA-immunoreactive terminals were visible throughout the SON of virgin and lactating rats. In such terminals, a variable number of electron-dense gold particles were seen over small (about 60-80 nm in diameter), clear vesicles, mitochondria and synaptic densities (Fig. 1). Gold particles were also visible in the cyloplasm and over microtubules in certain axonal profiles of the neuropil. All other cellular elements, including immediately adjacent terminals, showed little or no labeling (Fig. $1 B$ ). The GABA-positive terminals formed mainly symmetrical synaptic contact on dendritic (Fig. $1 A, B$ ) and somatic (Fig. $1 C$ ) profiles; some rare axoaxonic contacts were noted. GABA-immunoreactive terminals that made synaptic contact, in the same plane of section, onto two or more postsynaptic elements, somata and/or dendrites ("shared" synapses; Figs. $1 D$, 3) were visible in the SON of both groups.

Analysis of these sections revealed that the numerical density of all synapses (GABA positive or not) did not differ in the two groups and amounted to 35.3 and $34.5 \times 10^{6}$ synapses per $\mathrm{mm}^{3}$ of SON in virgin and lactating rats, respectively. In both groups, over one-third of all synapses showed GABA immunoreactivity; the density of axodendritic synapses was about double that of axosomatic synapses.

The surface area occupied by soma profiles increased from $47 \%$ of the total tissue area $\left(13,616 / 28,937 \mathrm{~mm}^{2}\right)$ in virgin rats to $55 \%\left(18,054 / 33,117 \mathrm{~mm}^{2}\right)$ in the lactating animals. After subtracting the soma surface area from the total surface area examined, the overall synaptic density in the neuropil of lactating rats was greater than that in virgin rats (Table 1), although this difference did not reach statistical significance. A statistically significant increase was apparent when the neurochemical identity of the synapses was taken into account and when we compared synaptic densities in the different portions of the SON. 

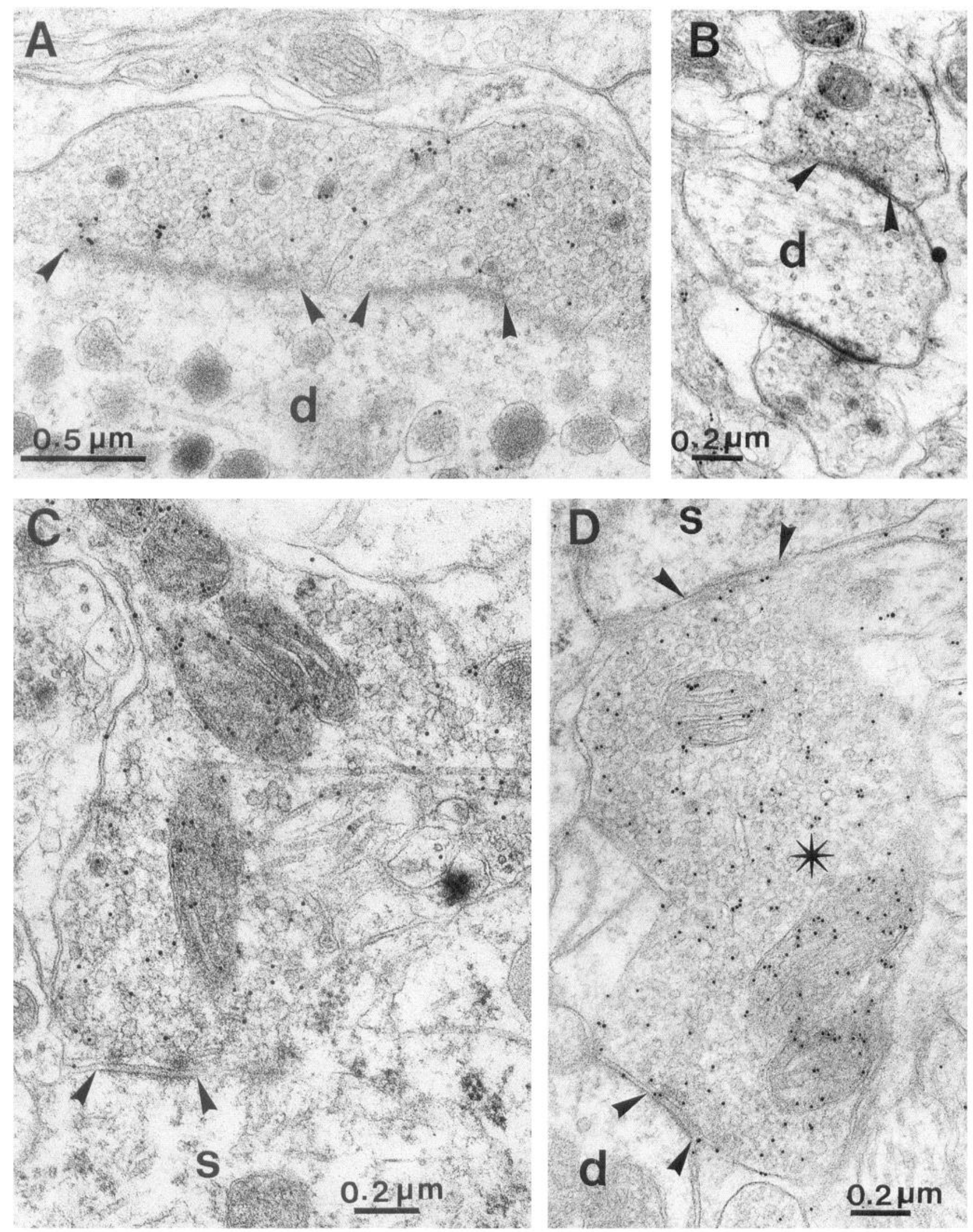

Figure 1. GABAergic terminals in the rat SON after single immunogold staining for GABA. They make symmetrical synaptic contact (between arrowheads) on dendrites ( $d$ in $A, B, D)$ and somata $(S$ in $C, D)$. Immunoreactivity is represented by colloidal gold particles present over small clear vesicles, mitochondria, and synaptic densities. In $D$, the terminal synapses simultaneously onto a soma and dendrite ("shared" synapse, ${ }^{*}$ ). 
Table 1. Number $\left(\times 10^{6}\right)$ synapses per $\mathrm{mm}^{3}$ neuropil $( \pm \mathrm{SD})$ in the SON of virgin and lactating rats

\begin{tabular}{llrlll} 
& \multicolumn{3}{l}{ Virgin } & \multicolumn{3}{l}{ Lactating } \\
\cline { 2 - 3 } \cline { 5 - 6 } Synapses & All & GABA+ & All & GABA+ \\
\hline Total & $\mathbf{6 6 . 6} \pm \mathbf{1 2 . 6}$ & $\mathbf{2 4 . 7} \pm \mathbf{5 . 3}$ & $\mathbf{7 5 . 8} \pm \mathbf{1 2 . 8}$ & $\mathbf{3 0 . 7} \pm \mathbf{7 . 1 *}$ \\
Axosomatic & $20.1 \pm 7.2$ & $\mathbf{7 . 8} \pm 2.8$ & $24.2 \pm 6.7$ & $9.6 \pm 4.3$ \\
Axodendritic & $46.5 \pm 8.4$ & $\mathbf{1 6 . 9} \pm 5.8$ & $51.6 \pm 11.7$ & $21.1 \pm 6.9$
\end{tabular}

*Significantly greater than corresponding value in virgin rats, $p \leq 0.05$, MannWhitney $U$ test.

Thus, the density of GABA-positive synapses was greater in lactating rats, an increase that implicated both axosomatic and axodendritic synapses (Table 1). Moreover, such an increase was particularly apparent in the mid and posterior portions of the SON, especially for axosomatic synapses (Table 2). As for the density of GABA-negative axosomatic or axodendritic synapses, it did not differ in the two groups, whether we considered it as a whole or in relation to the different portions of the SON (Tables 1, 2).

In agreement with earlier studies (Theodosis et al., 1981; Hatton and Tweedle, 1982; Theodosis and Poulain, 1984; Tweedle and Hatton, 1984), "shared" synapses (Figs. 1D,3) were visible in the nuclei of both groups although they were not very abundant. Nevertheless, their incidence did increase in the SON of lactating animals. Thus, 25 terminals making synaptic contact onto two or more postsynaptic elements in the same plane of section were detected in the lactating group, coupling somata and/or dendrites and of these 13 were GABA immunoreactive. We counted 14 in the virgin group, 6 of which were GABA positive.

\section{Double immunostaining for $G A B A$ and $O T-N p$}

In sections stained simultaneously for GABA and OT-Np, the intensity and localization of GABA immunoreactivity (represented by $10 \mathrm{~nm}$ colloidal gold particles) were similar to those after single GABA staining. OT-Np immunoreactivity (represented by $5 \mathrm{~nm}$ colloidal gold particles) was restricted to membrane-bound secretory granules in the cytoplasm of certain neurosecretory somata (Figs. 2, 3) and dendrites. The OT-Nppositive profiles were visible together with other neurosecretory profiles that were completely devoid of immunoreactivity. We can reasonably assume that most of the latter were vasopres- sinergic since several analyses have established that the rat $\mathrm{SON}$, especially in its mid-portion, contains oxytocinergic and vasopressinergic neurons in about equal proportions (see, e.g., Rhodes et al., 1981; Theodosis et al., 1986a).

GABA-immunoreactive terminals synapsed both on OT-Nppositive (Figs. 2, 3) and OT-Np-negative profiles. Analysis of these double-stained sections revealed that half of all synapses on OT-Np-positive somata in lactating rats were GABAergic, a proportion significantly greater than that recorded in virgin rats (Fig. 4). On the other hand, the proportion of GABAergic synapses on OT-Np-negative somata did not differ significantly between virgin and lactating rats (Fig. 4).

\section{Discussion}

Density of $G A B A$ synapses in the rat SON

To our knowledge, there has been only one other study that analyzed the synaptic density of the rat SON (Leranth et al., 1975), and it is noteworthy that, even though the quantitative methods used differ, the values obtained here are quite similar to those reported earlier, showing an overall numerical density of about $35 \times 10^{6}$ synapses per $\mathrm{mm}^{3}$ of tissue. From numerous immunocytochemical studies at the electron microscopic level, it is now clear that SON synapses contain most neurotransmitters found in other central systems, notably GABA (Van Den Pol, 1985; Theodosis et al., 1986c; Buijs et al., 1987; Decavel and Van Den Pol, 1990) and glutamate (Meeker et al., 1989; Van Den Pol et al., 1990; Decavel and Van Den Pol, 1992), and to a lesser extent, the catecholamines (Sawchenko and Swanson, 1982; Sawchenko et al., 1983; Buijs et al., 1984; Decavel et al., 1987).

The present observations indicate that over one-third of all synapses in the SON, at least of virgin rats, are GABAergic. This is less than the proportion estimated in our earlier study, which was performed on semithin sections examined at the light microscope level after staining with a postembedding technique for GAD, the biosynthetic enzyme for GABA (Theodosis et al., 1986c). However, in that study, the counted immunoreactive punctae represented all immunoreactive boutons and even cut axonal profiles, while in the present study only those terminals showing synaptic contacts were taken into account. In agreement with earlier studies (Theodosis et al., 1986c; Decavel and Van Den Pol, 1990), the GABAcrgic terminals were seen to make symmetrical synaptic contact on dendrites and somata. We es-

Table 2. Comparison of synaptic density in the anterior, mid, and posterior SON of virgin and lactating rats

\begin{tabular}{|c|c|c|c|c|c|}
\hline & \multirow[b]{2}{*}{ Synapses } & \multicolumn{2}{|l|}{ Virgin } & \multicolumn{2}{|l|}{ Lactating } \\
\hline & & All & GABA+ & All & GABA+ \\
\hline \multirow[t]{3}{*}{ Anterior } & Total & $72.5 \pm 15.1$ & $23.3 \pm 5.7$ & $68.6 \pm 8.7$ & $21.9 \pm 5.3$ \\
\hline & Axosomatic & $23.0 \pm 10.2$ & $8.6 \pm 3.8$ & $19.3 \pm 5.2$ & $5.4 \pm 3.2$ \\
\hline & Axodendritic & $54.4 \pm 6.5$ & $18.1 \pm 4.9$ & $49.4 \pm 13.2$ & $19.6 \pm 6.4$ \\
\hline \multirow[t]{3}{*}{ Medial } & Total & $58.6 \pm 8.5$ & $20.5 \pm 7.1$ & $77.6 \pm 13.2$ & $28.9 \pm 3.1^{*}$ \\
\hline & Axosomatic & $19.8 \pm 4.4$ & $8.6 \pm 1.4$ & $23.1 \pm 5.7$ & $10.1 \pm 1.6^{* *}$ \\
\hline & Axodendritic & $38.8 \pm 7.9$ & $11.9 \pm 6.4$ & $54.4 \pm 7.4$ & $18.8 \pm 2.7$ \\
\hline \multirow[t]{3}{*}{ Posterior } & Total & $63.8 \pm 7.0$ & $26.6 \pm 0.9$ & $78.3 \pm 18.1$ & $36.9 \pm 7.3^{*}$ \\
\hline & Axosomatic & $16.4 \perp 7.4$ & $5.7 \pm 2.4$ & $29.2 \perp 6.9$ & $13.2 \pm 3.9^{* *}$ \\
\hline & Axodendritic & $47.5 \pm 0.5$ & $20.9 \pm 3.1$ & $49.0 \pm 17.1$ & $23.7 \pm 11.2$ \\
\hline
\end{tabular}

Data are presented as mean number $\left(\times 10^{6}\right)$ synapses per $\mathrm{mm}^{3}$ neuropil $\pm \mathrm{SD}^{*}$ and **, significantly greater than corresponding value in virgin rats at $p \leq 0.05$ and $p \leq 0.01$, Mann-Whitney $U$ test. 

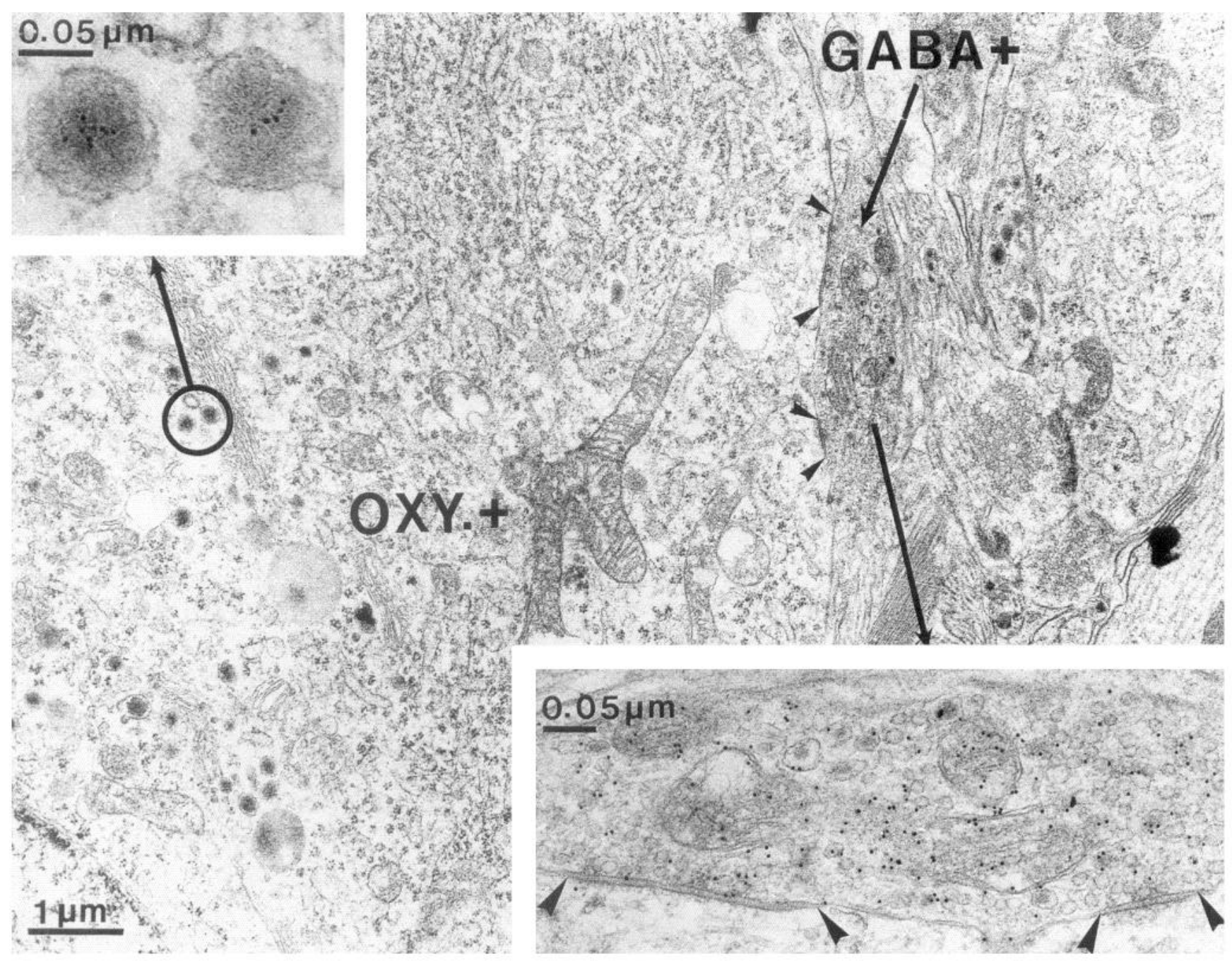

Figure 2. Simultaneous dual immunogold staining for GABA and OT-Np. A GABAergic terminal $(G A B A+)$ is seen to make synaptic contact (between arrowheads, and at higher magnification in the inset, lower right) onto an oxytocinergic $(O X Y+)$ soma. The former is identified by 10 $\mathrm{nm}$ colloidal gold particles over its small clear vesicles and mitochondria, and the latter by $5 \mathrm{~nm}$ colloidal gold particles over its secretory granules (circle, and at higher magnification in the inset, upper left).

timated that the density of axodendritic contacts was double that of axosomatic contacts.

We feel reasonably confident in the validity of our present analyses since they were carried out on ultrathin sections immunostained with a postembedding technique that allows reliable estimation of immunoreactive profiles. After such a procedure, ultrastructure is well preserved and synapses are readily identifiable. It is also a surface reaction of sections and the immunosignal is not dependent on the penetration of immunoreagents into the preparation, which means that we can reliably identify not only immunopositive but also immunonegative profiles (for further discussion, see Theodosis et al., 1986a; Merighi et al., 1989). In addition, such a procedure allows staining of both pre- and postsynaptic partners on the same section. We were thus able to see that GABA afferents to the SON indeed impinge on both oxytocin and presumptive vasopressin neurons, and in unstimulated animals, to about an equivalent degree. However, we may have underestimated the number of immunoreactive profiles. As in all analyses of material stained immunocytochemically, profiles containing small amounts of GABA (because of differences in the metabolism, release, and/ or uptake of the transmitter) would have had undetectable levels of GABA immunoreactivity; for oxytocinergic somata, if the profile did not display any secretory granules in the plane of section examined, its identity would be left open to question. Nevertheless, the aim of this study was to arrive at a comparative appreciation of the examined profiles, and we assumed that such an underestimation probably applied to all the areas examined regardless of their origin in relation to the different parts of the nucleus or in relation to the physiological state of the animal. This assumption does not appear too unreasonable when one considers that such differences in GABA immunoreactivity have not been described in any of the numerous systems in which this transmitter system has been examined. Regarding the somatic profiles, this and earlier studies (see, e.g., Theodosis et al., 1986a) have shown that they continue to display secretory granules even under sustained stimulation.

The origin of the GABAergic input to SON neurons remains unknown. It is not intranuclear since no GABAergic cell bodies were detected in the nucleus, in this or in earlier studies (Theodosis et al., 1986c). One source may be perinuclear since several neuroanatomical tracing studies have indicated that much of 

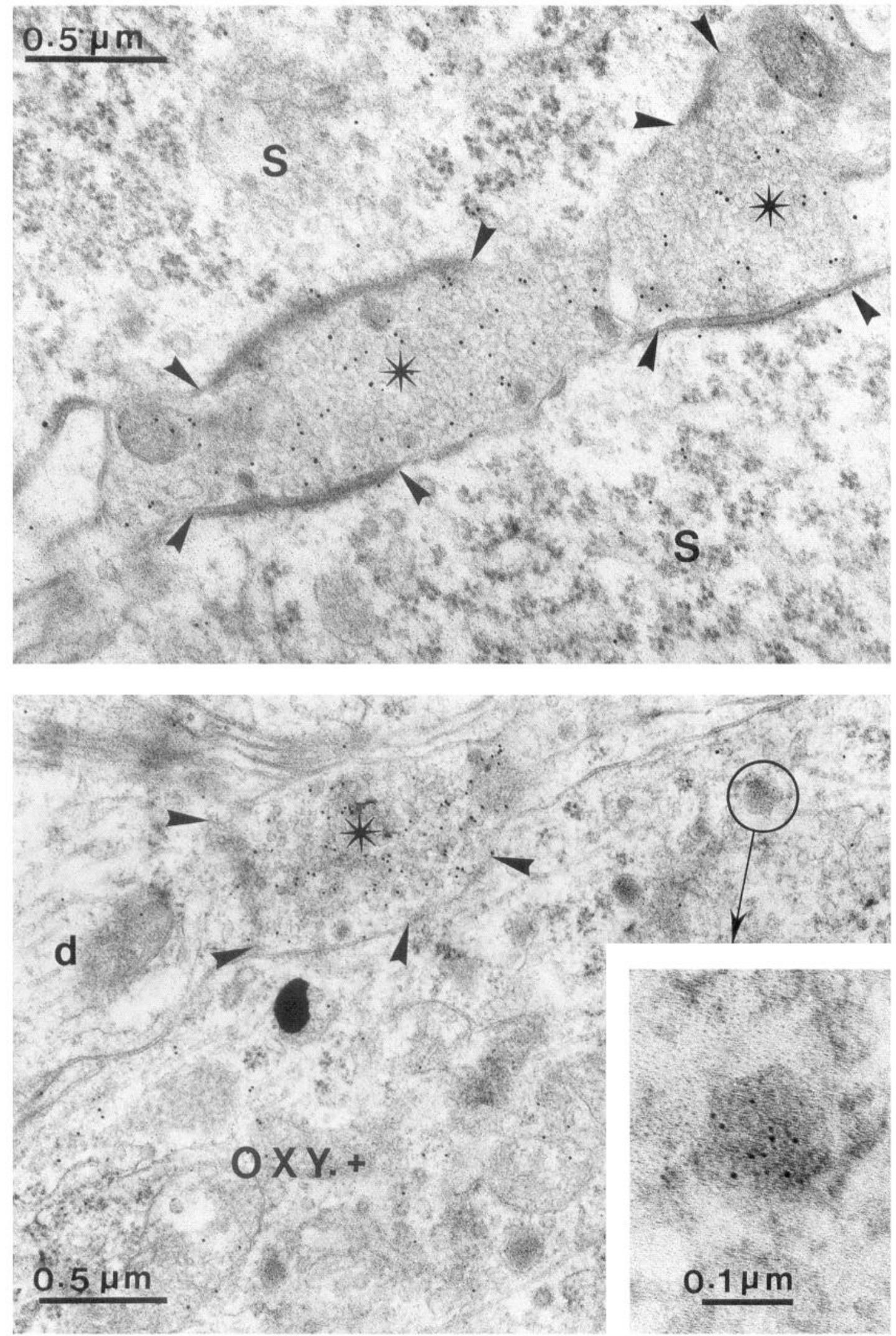

Figure 3. "Shared" GABAergic synapses in the SON of lactating rats. The top panel shows two terminals (asterisks) making synaptic contact (between arrowheads) simultaneously onto two somata $(S)$. They display GABA immunoreactivity $(10 \mathrm{~nm}$ gold particles) after single immunostaining for GABA. The bottom panel displays one GABAergic terminal (asterisk) making synaptic contact (between arrowheads) both onto a dendrite (d) and a soma. Double immunostaining on the same ultrathin section permitted identification of the latter as oxytocinergic $(O X Y+)$, attested by the presence of $5 \mathrm{~nm}$ gold particles over its secretory granules (circle and at higher magnification in the inset). 
the direct synaptic input to the SON originates from the adjacent hypothalamic perinuclear zone (Zaborszky et al., 1975; Tribollet et al., 1985). In our earlier studies, we did detect GAD- and GABA-positive cell bodies in this area (Theodosis et al., 1986c). A more recent study has confirmed these earlier data, by combining neuroanatomical tracing and GAD immunocytochcmistry (Roland and Sawchenko, 1993). Nevertheless, the projections from local sources appear scarce (Roland and Sawchenko, 1993). The direct GABAergic input to the nucleus probably derives from various sources, including areas such as the diagonal band of Broca (Jhamandas et al., 1989).

\section{GABA and the synaptic plasticity of the SON}

It has been known for several years that lactation induces striking changes in the conformation of magnocellular oxytocinergic neurons and their astrocytes (for review, see Theodosis and Poulain, 1987, 1993). Thus, in lactating rats, astrocytic coverage of oxytocinergic neurons diminishes and over $80 \%$ of all their somata and dendrites in the rat SON and PVN become directly juxtaposed; after weaning, there are again few directly juxtaposed neuronal elements in these nuclei. Concomitant with these ncuronal-glial changes, one always sees changes in the synaptic input onto the neurons.

Until now, the most obvious manifestation of this synaptic plasticity has been changes in the incidence of "shared" synapses, which increase in frequency already at parturition and diminish again after weaning (Theodosis et al., 1981; Hatton and Tweedle, 1982; Theodosis and Poulain, 1984; Montagnese et al., 1987). The present observations confirm this increased frequency of "shared" synapses in the nuclei of lactating animals. Nevertheless, their incidence, which in this and earlier studies was appreciated simply by counting the number of terminals making such synaptic contacts in randomly chosen sections, was relatively low. Such counts probably greatly underestimate their frequency; analysis on serial sections would certainly provide a better evaluation of their occurrence and contribution to synaptic remodeling of the nucleus.

What the present analyses reveal is that synaptic plasticity in the rat SON may be even more pronounced than we thought previously. Not only "shared" synapses but also terminals making single synaptic contact appear to increase in frequency in response to lactation. Two main observations support such a contention. First, although somata and processes of SON neurons hypertrophy during lactation (see also Kalimo, 1975; Theodosis et al., 1986a; Salm et al., 1988; Modney and Hatton, 1989), the overall synaptic density in the nucleus in lactating rats did not diminish but remained similar to that in virgin rats. Second, an increased numerical density of synapses was visible when we evaluated the number of synapses in terms of the neuropil (by subtracting the proportion of sampled areas occupied by soma profiles). It may be argued that during lactation there is shrinking of the neuropil, especially in view of the neuronal-glial changes cited earlier. Nevertheless, we think this is not the case since earlier analyses have shown that dendritic and axonal surface areas increase during lactation (Kalimo, 1975; Salm et al., 1988), as do somatic areas. An increase in the overall neuropil surface area, at least in the ventral portions of the nucleus, has also been documented (Salm et al., 1988). We also believe that there is no shrinking of the other major element of the SON neuropil, its glial cells, in spite of the increased incidence of directly juxtaposed neuronal processes. Several different analyses have now indicated that astrocytic coverage of magnocellular somata ac-

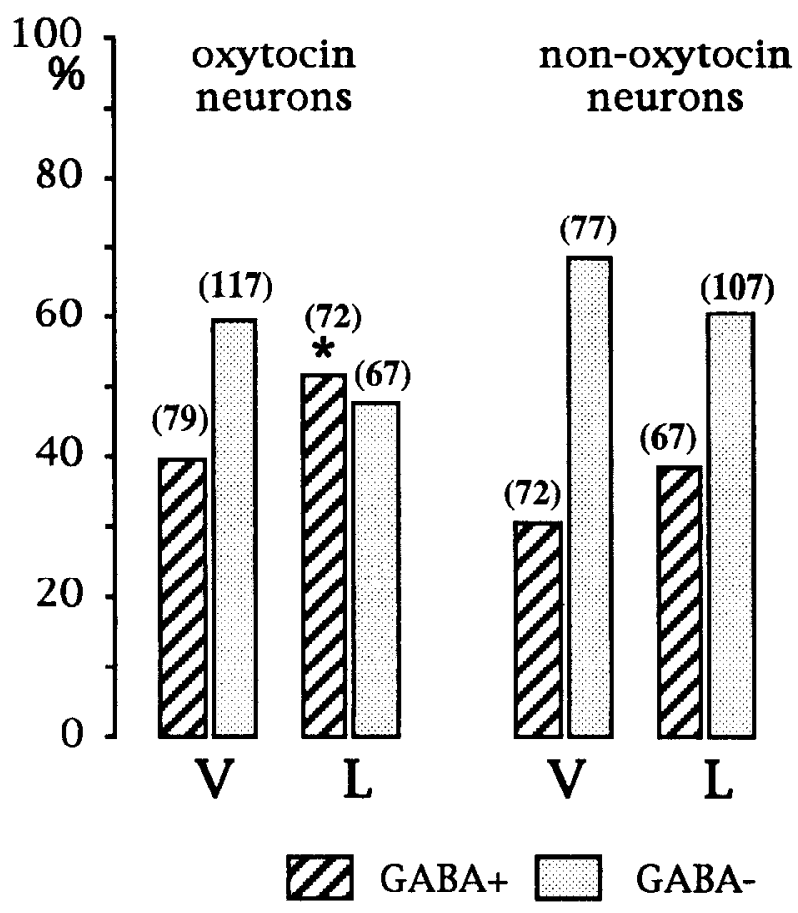

Figure 4. Distribution of GABA-immunoreactive synapses on oxytocinergic and non-oxytocinergic (presumptive vasopressinergic) somata in the SON of virgin $(V)$ and lactating $(L)$ rats. Note that GABAergic synapses were more numerous on oxytocinergic profiles in lactating rats $\left(^{*}, p<0.025, \chi^{2}\right.$ analysis on raw data). The proportion of GABA-positive synapses on non-oxytocinergic profiles was similar in the two groups and no different from the proportion on oxytocinergic profiles in virgin rats. Numbers in parentheses correspond to the number of synapses analyzed in each group.

tually increases in absolute values to meet their hypertrophy under stimulation (Chapman et al., 1986; Theodosis et al., 1986a; Modney and Hatton, 1989); what is peculiar to oxytocinergic somata is that the proportion of their plasmalemma covered by astrocytic processes diminishes (Chapman et al., 1986; Theodosis et al., 1986a). These observations thus indicate that there is a rearrangement of astrocytic processes in this nucleus during stimulation and not necessarily a shrinking. As for astrocytic cell bodies, very few occur in the nucleus proper, most being present along the base of the brain (Bonfanti et al., 1993).

The increase in the numerical density of synapses in the SON ncuropil during lactation became even more evident when we took into account the neurochemical identity of the synapses and the different parts of the nucleus. We were thus able to see that the density of GABA-immunoreactive synapses, especially those impinging on somata, was significantly greater in lactating rats, an increase that appeared most obvious in the mid and posterior portions of the nucleus. On the other hand, no regional differences were apparent in the density of GABA-negative synapses throughout the nuclei of virgin or lactating animals. It may be argued that this increase reflects a functional rather than structural change whereby more GABA neurons would express more neurotransmitter at their terminals during lactation. Although we cannot discount this possibility, we do believe, for the reasons cited above, that there is structural synaptic plasticity in this nucleus during lactation, revealed not only by augmented synaptic densities but also by the increase in terminals contributing to "shared" synapses, at least half of which displayed GABA immunoreactivity. 
On the other hand, why an increased density of GABAergic synapses at lactation is particularly visible in the mid and posterior SON remains to be determined. It is probably not due to a special disposition of the two neurosecretory populations in the nucleus since light and electron microscopic analyses show that oxytocinergic neurons occur intermingled with vasopressin neurons throughout the SON (Rhodes et al., 1981; Theodosis et al., 1986a). It may be that the source of GABAergic afferents differs according to the regions that they innervate.

\section{$G A B A$ and oxytocin neurons}

Analysis of double-stained sections confirmed our carlier observations (Theodosis et al., 1986c) indicating that an important proportion of all synapses on both oxytocin and vasopressin neurons is GABAergic. Moreover, they confirm our earlier suggestion that the synaptic plasticity of the SON affects GABAergic synapses on oxytocin neurons: the proportion of GABAergic synapses on oxytocinergic profiles significantly increased in lactating animals while it was similar on presumptive vasopressinergic profiles in virgin and lactating rats.

Oxytocinergic neurons display a particular electrical activation during lactation that is characterized by intermittent highfrequency discharges of action potentials occurring synchronously throughout the population and resulting in a bolus release of hormone and reflex milk ejection (for review, see Poulain and Wakerley, 1982). As in other neuronal systems, there is good evidence that GABA inhibits the electrical activity of oxytocin neurons (Randle et al., 1986; Randle and Renaud, 1987) and the milk ejection reflex (Voisin et al., 1992). It therefore appears contradictory that oxytocin neurons receive an enhanced inhibitory input during lactation. It may be that an augmentation in GABAergic input to oxytocinergic somata is a mere compensatory mechanism to meet their hypertrophy during lactation, providing an equivalent degree of inhibition as that acting in virgin rats. Another possibility is that a strong inhibition is necessary to facilitate further their synchronous activation induced by an excitatory stimulus acting only at particular times, as, for example, just before reflex milk ejection. Finally, it may also be that a further inhibition is exerted on oxytocin neurons to prevent them from being stimulated by factors other than those necessary for lactation. For instance, there is evidence that the oxytocin system in lactating animals is less reactive to osmotic (Hartman et al., 1987) and stressful (Lightman and Young, 1989) stimuli. In addition, c-fos expression is markedly reduced in oxytocin neurons of dehydrated or hemorrhaged lactating rats (Fenelon et al., 1993).

\section{Concluding remarks}

The SON is certainly not the only neuronal system in the adult CNS that displays a transmitter-related remodeling of synapses. Such a phenomenon has been described in structures as different as the red nucleus, where sprouting of GABAergic synapses occurs after lesions of the nucleus interpositus (Katsumaru et al., 1986), and visual cortex, where the richness of the environment modifies the excitatory and inhibitory equilibrium through a restructuring of inhibitory inputs (Beaulieu and Colonnier, 1987). Another hypothalamic center that shows activity-linked synaptic plasticity of a GABA input is the arcuate nucleus (Parducz et al., 1993). At present we are far from understanding the cellular mechanisms underlying this kind of synaptic plasticity, but the involvement of particular afferents highlights the participation of both pre- and postsynaptic partners.

In the magnocellular nuclei, as in certain other hypothalamic (Olmos et al., 1989; Witkin et al., 1991; Parducz et al., 1993) and peripheral systems (Purves et al., 1987), remodeling of synaptic inputs occurs in concert with neuronal-glial conformational changes. Studies from the developing nervous system indicate that formation and stabilization of synaptic connections require not only interactions between pre- and postsynaptic partners but also interactions between these and the astrocytes with which they are associated. Such interaction may also be important in adult synaptic rearrangements. In the hypothalamic nuclei, we still do not know whether the changing incidence of synapses represents de novo formation of axonal terminals with consequent degeneration or modification of preexisting ones such that synaptic specializations appear and disappear during different physiological conditions. That neither growth cones nor degenerating terminals have been detected in the hypothalamic centers would argue in favor of the latter hypothesis, suggesting that the number of boutons remains the same but there is creation of new active sites concomitant with the appearance and disappearance of intervening glial processes (for further discussion, see Theodosis and Poulain, 1993).

\section{References}

Beaulieu C, Colonnier M (1987) Effect of the richness of the environment on the cat visual cortex. J Comp Neurol 266:478-494.

Ben-Barak Y, Russell JT, Whitnall MH, Ozato K, Gainer H (1985) Neurophysin in the hypothalamo-neurohypophysial system. I. Production and characterization of monoclonal antibodies. J Neurosci 5:81-97.

Bonfanti L, Poulain DA, Theodosis DT (1993) Radial glia-like cells in the supraoptic nucleus of the adult rat. J Neuroendocrinol 5:1-6.

Buijs RM, Geffard M, Pool CW, Hoorneman EMD (1984) The dopaminergic innervation of the supraoptic and paraventricular nucleus. A light and electron microscopical study. Brain Res 323:65-72.

Buijs RM, Van Vulpen EHS, Geffard M (1987) Ultrastructural localization of GABA in the supraoptic nucleus and neural lobe. Neuroscience 20:347-355.

Chapman DB, Theodosis DT, Montagnese C, Poulain DA, Morris JF (1986) Osmotic stimulation causes structural plasticity of neuroneglia relationships of the oxytocin but not vasopressin secreting neurones in the hypothalamic supraoptic nucleus. Neuroscience 17:679686.

Colonnier M, Beaulieu C (1985) An empirical assessment of stereological formulae applied to the counting of synaptic disks in the cerebral cortex. J Comp Neurol 231:175-179.

Decavel C, Van Den Pol AN (1990) GABA: a dominant neurotransmitter in the hypothalamus. J Comp Neurol 302:1019-1037.

Decavel C, Van Den Pol AN (1992) Converging GABA- and glutamate-immunoreactive axons make synaptic contact with identified hypothalamic neurosecretory neurons. J Comp Neurol 316:104-116.

Decavel C, Geffard M, Calas A (1987) Comparative study of dopamine- and noradrenaline-immunoreactive terminals in the paraventricular and supraoptic nuclei of the rat. Neurosci Lett 77:149-154.

Fenelon VS, Poulain DA, Theodosis DT (1993) Oxytocin neuron activation and Fos expression: a quantitative immunocytochemical analysis of the effect of lactation, parturition, osmotic and cardiovascular stimulation. Neuroscience 53:77-89.

Hartman RD, Rosella-Dampman LM, Summy-Long JY (1987) Endogenous opioid peptides inhibit oxytocin release in the lactating rat after dehydration and urethane. Endocrinology 121:536-543.

Hatton GI (1990) Emerging concepts of structure-function dynamics in adult brain: the hypothalamo-neurohypophysial system. Prog Neurobiol 34:437-504.

Hatton GI, Tweedle CD (1982) Magnocellular peptidergic neurons in hypothalamus: increases in membrane apposition and number of specialized synapses from pregnancy to lactation. Brain Res Bull 8:197204.

Jhamandas JH, Raby W, Rogers J, Buijs RM, Renaud LP (1989) Di- 
agonal band projection towards the hypothalamic supraoptic nucleus: light and electron microscopic observations in the rat. J Comp Neurol 282:15-23.

Kalimo H (1975) Ultrastructural studies on the hypothalamic neurosecretory neurons of the rat. III. Paraventricular and supraoptic neurons during lactation and dehydration. Cell Tissue Res 163:151168.

Katsumaru H, Murakami F, Wu J-Y, Tsukahara N (1986) Sprouting of GABAergic synapses in the red nucleus after lesions of the nucleus interpositus in the cat. J Neurosci 6:2864-2874.

Leranth Cs, Zaborszky L, Marton J, Palkovits M (1975) Quantitative studies on the supraoptic nucleus in the rat. I. Synaptic organization. Exp Brain Res 22:509-523.

Lightman SL, Young WS III (1989) Lactation inhibits stress mediated secretion of corticosterone and oxytocin and hypothalamic accumulation of CRF and enkephalin messenger ribonucleic acids. Endocrinology 124:2358-2364.

Meeker RB, Swanson DJ, Hayward JN (1989) Light and electron microscopic localization of glutamate immunoreactivity in the supraoptic nucleus of the rat hypothalamus. Neuroscience 33:157-167.

Merighi A, Polak JM, Fumagalli G, Theodosis DT (1989) Ultrastructural localisation of neuropeptides and GABA in the rat dorsal horn a comparison of different immunogold labelling techniques. J Histochem Cytochem 37:529-540.

Modney BK, Hatton GI (1989) Multiple synapse formation: a possible compensatory mechanism for increased cell size in rat supraoptic nucleus. J Neuroendocrinol 1:21-27.

Montagnese C, Poulain DA, Vincent JD, Theodosis DT (1987) Structural plasticity in the rat supraoptic nucleus during gestation, postpartum lactation and suckling-induced pseudogestation and lactation. J Endocrinol 115:97-105.

Olmos G, Naftolin F, Perez J, Tranque PA, Garcia-Segura LM (1989) Synaptic remodeling in the rat arcuate nucleus during the estrous cycle. Neuroscience 32:663-667.

Parducz A, Perez J, Garcia-Segura LM (1993) Estradiol induces plasticity of GABAergic synapses in the hypothalamus. Neuroscience 53: 395-402.

Paxinos G, Watson C (1986) The rat brain in stercotaxic coordinates. San Diego: Academic.

Poulain DA, Wakerley JB (1982) Electrophysiology of hypothalamic magnocellular neurones secreting oxytocin and vasopressin. Neuroscience 7:773-808.

Purves D, Voyvodic JT, Magrassi L, Yawo H (1987) Nerve terminal remodeling visualized in living mice by repeated examination of the same neuron. Science 238:1122-1126.

Randle JCR, Renaud LP (1987) Actions of $\gamma$-aminobutyric acid on rat supraoptic nucleus neurosecretory neurones in vitro. J Physiol (Lond) 387:629-647.

Randle JCR, Bourque CWB, Renaud LP (1986) Characterization of spontaneous and evoked inhibitory postsynaptic potentials in rat supraoptic neurosecretory neurons in vitro. J Neurophysiol 56:17031717.

Rhodes CH, Morrell JI, Pfaff DW (1981) Immunohistochemical analysis of magnocellular elements in rat hypothalamus: distribution and number of cells containing neurophysin, oxytocin and vasopressin. J Comp Neurol 198:45-64.

Roland BL, Sawchenko PE (1993) Local origins of some GABAergic projections to the paraventricular and supraoptic nuclei of the hypothalamus in the rat. J Comp Neurol 332:123-143.

Salm AK, Modney BK, Hatton GI (1988) Alterations in supraoptic nucleus ultrastructure of maternally behaving virgin rats. Brain Res Bull 21:685-691.

Sawchenko PE, Swanson LW (1982) The organization of noradren- ergic pathways from the brainstem to the paraventricular and supraoptic nuclei in the rat. Brain Res Rev 4:275-325.

SawchenkoPE,SwansonLW,SteinbuschHWM,VerhofstadAAJ (1983)The distribution and cells of origin of serotonergic inputs to the paraventricular and supraoptic nuclei of the rat. Brain Res 277:355-360.

Seguela P, Geffard M, Buijs PM, Le Moal M (1984) Antibodies against gamma-aminobutyric acid: specificity studies and immunocytochemical results. Proc Natl Acad Sci USA 81:3888-3892.

Theodosis DT, Poulain DA (1984) Evidence for structural plasticity in the supraoptic nucleus of the rat hypothalamus in relation to gestation and lactation. Neuroscience 11:183-193.

Theodosis DT, Poulain DA (1987) Oxytocin-secreting neurones: a physiological model for structural plasticity in the adult mammalian brain. Trends Neurosci 10:426-430.

Theodosis DT, Poulain DA (1989) Neuronal-glial and synaptic plasticity in the adult rat paraventricular nucleus. Brain Res 484:36 I366.

Theodosis DT, Poulain DA (1993) Activity-dependent neuronal-glial and synaptic plasticity in the adult mammalian hypothalamus. Neuroscience 57:501-535.

Theodosis DT, Poulain DA, Vincent JD (1981) Possible morphological bases for synchronisation of neuronal firing in the rat supraoptic nucleus during lactation. Neuroscience 6:919-929.

Theodosis DT, Chapman DB, Montagnese C, Poulain DA, Morris JF (1986a) Structural plasticity in the hypothalamic supraoptic nucleus at lactation affects oxytocin- but not vasopressin-secreting neurones. Neuroscience 17:661-678.

Theodosis DT, Montagnese C, Rodriguez F, Vincent JD, Poulain DA (1986b) Oxytocin induces morphological plasticity in the adult hypothalamo-neurohypophysial system. Nature 322:738-740.

Theodosis DT, Paut L, Tappaz ML (1986c) Immunocytochemical analysis of the GABAergic innervation of oxytocin- and vasopressinsecreting neurones in the rat supraoptic nucleus. Neuroscience 19: 207-222.

Tribollet E, Armstrong WE, Dubois-Dauphin M, Dreifuss JJ (1985) Extra-hypothalamic afferent inputs to the supraoptic nucleus area of the rat as determined by retrograde and anterograde tracing techniques. Neuroscience 15:135-148.

Tweedle CD, Hatton GI (1984) Synapse formation and disappearance in adult rat supraoptic nucleus during different hydration states. Brain Res 309:373-376.

Van Den Pol AN (1985) Dual ultrastructural localization of two neurotransmitter-related antigens: colloidal gold-labeled neurophysin-immunoreactive supraoptic neurons receive peroxidase-labeled glutamate decarboxylase- or gold-labeled GABA-immunoreactive synapses. J Neurosci 5:2940-2954.

Van Den Pol AN, Wuarin J-P, Dudek FE (1990) Glutamate, the dominant excitatory transmitter in neuroendocrine regulation. Science 250:1276-1278.

Voisin D, Herbison AE, Poulain DA (1992) Intracerebroventricular injection of muscimol or bicuculline inhibits the milk ejection reflex in the rat. J Physiol (Lond) 452:211P.

Weibel ER, Bolender RP (1973) Stereological techniques for electron microscopic morphometry. In: Principles and techniques of electron microscopy: biological applications (Hayat MA, ed), pp 239-295. New York: Van Nostrand Reinhold.

Witkin JW, Ferin M, Popilskis SJ, Silverman A-J (1991) Effects of gonadal steroids on the ultrastructure of GnRH neurons in the rhesus monkey: synaptic input and glial apposition. Endocrinology 129:10831092.

Zaborszky L, Leranth Cs, Makara GB, Palkovits M (1975) Quantitative studies on the supraoptic nucleus in the rat. II. Afferent fiber connections. Exp Brain Res 22:525-540. 